\title{
Dimensiones Comunes a las Atribuciones de Pobreza y Riqueza
}

\section{Common Dimensions for Poverty and Wealth Attributions}

\author{
Juan Carlos Castillo y Mauricio Rivera-Gutiérrez \\ Pontificia Universidad Católica de Chile
}

\begin{abstract}
La teoría de la atribución posee una larga tradición en el estudio de las explicaciones que las personas otorgan al origen de la pobreza. Sin embargo, la investigación sobre las causas percibidas de la riqueza han tenido hasta ahora un lugar secundario y no relacionado con las atribuciones de pobreza. Este artículo se centra en la relación de las atribuciones de riqueza y pobreza desde una perspectiva de medición de variables latentes, considerando dos tipos básicos de atribuciones: internas (conducta individual) y externas (determinantes socioestructurales). Se analizaron las respuestas a una escala de 10 indicadores de la encuesta Justicia Social y Participación Ciudadana, aplicada a una muestra probabilística estratificada por conglomerados en Chile el año $2013(N=1245)$. Basados en evidencia previa, se estimó un modelo factorial confirmatorio de atribuciones internas y externas, tanto para pobreza como riqueza (cuatro factores), en base al cual se estima un modelo de segundo orden de dos factores, uno de atribuciones internas y otro de atribuciones externas. Los resultados entregan por primera vez evidencia de atribuciones comunes para la riqueza y la pobreza.
\end{abstract}

Palabras clave: atribuciones, de pobreza, atribuciones de riqueza, atribuciones internas, atribuciones externas, análisis factorial confirmatorio

The theory of attributions has long been used to study people's explanations for the origin of poverty. Nevertheless, research on the perceived causes of wealth has thus far been sidelined and isolated from the study of poverty attributions. This article focuses on the relationship between poverty and wealth attributions from a latent variable measurement perspective. To examine this relationship, two basic attribution types were considered: internal (based on individual behavior) and external (based on socio-structural determinants). Answers to a 10indicator scale from the Social Justice and Citizenship Participation survey were analyzed. The instrument was administered to a stratified cluster probability sample in Chile in $2013(N=1245)$. Based on prior evidence, a confirmatory factor model of internal and external poverty and wealth attributions was estimated (four factors). Based on it, a second-order two-factor model was estimated, one of internal and another of external attributions. For the first time results yield evidence of common attributions for poverty and wealth.

Keywords: poverty attributions, wealth attributions, internal attributions, external attributions, confirmatory factor analysis

Las maneras en que los individuos explican las causas de la pobreza y la riqueza en las sociedades modernas constituyen un foco de interés para la teoría de las atribuciones en psicología social y para las ciencias sociales en general. En la medida que los pobres son considerados responsables de su situación (i.e., atribución interna), sería menos probable que exista cualquier intención o motivación por modificar las condiciones que permiten la existencia de la pobreza. Los posibles impactos de esta forma de explicar el origen de la pobreza podrían reflejarse en las actitudes hacia ricos y pobres (Kluegel, Csepeli, Kolosi, Örkény \& Neményi, 1995; Sigelman, 2012), el grado en que las desigualdades de ingreso se perciben como justas (Schneider \& Castillo, 2015) y la percepción general de las desigualdades sociales (Bullock \& Limbert, 2003). Tal como plantean Lepianka, van Oorschot y Gelissen (2009), el estudio de las atribuciones de la riqueza y la pobreza podría "proveer un entendimiento importante sobre la legitimación de las desigualdades sociales y económicas" (p. 422).

En el estudio de las atribuciones de riqueza y pobreza es posible encontrar dos principales desafíos. El primero de ellos dice relación con la conceptualización de las posibles formas de atribución y la relación entre

Juan Carlos Castillo, Instituto de Sociología, Pontificia Universidad Católica de Chile, Santiago, Chile; Mauricio RiveraGutiérrez, Centro de Medición MIDE UC, Pontificia Universidad Católica de Chile, Santiago, Chile.

Esta investigación ha contado con financiamiento del proyecto FONDECYT 11121203, FONDECYT 1160921 y de CONICYT/FONDAP/15130009, de los cuales el primer autor es investigador principal.

La correspondencia relativa a este artículo debe ser dirigida a Juan Carlos Castillo, Instituto de Sociología, Pontificia Universidad Católica de Chile, Avda. Vicuña Mackenna 4860, Macul, Santiago, Chile. E-mail: jcastillov@uc.cl 
ellas. Si bien tradicionalmente se identifican atribuciones de tipo interno (i.e., responsabilizando al individuo) y externo (i.e., responsabilizando a la sociedad), no existe claridad respecto de la relación entre atribuciones internas y externas de fenómenos contrapuestos, como son la riqueza y la pobreza. De esta manera, cabría hacer la pregunta respecto de si las atribuciones internas de riqueza y pobreza se encuentran relacionadas, así como también las externas. Concretamente, una persona que atribuye internamente (o externamente) la pobreza ¿piensa lo mismo sobre la riqueza? La respuesta a esta pregunta permitiría ahondar en patrones más generales de atribución y en sus potenciales consecuencias tanto metodológicas como conceptuales. El segundo desafío en la investigación en atribuciones, y que se conecta con el primero, es el de la medición. Si bien hay ciertos indicadores usados reiteradamente en distintos estudios de atribución desde su formulación inicial en los años 70, no existe una escala consensuada y, además, hay poca disponibilidad de datos conjuntos de atribuciones de riqueza y de pobreza que permitan realizar un análisis confirmatorio de la estructura de las dimensiones latentes a ambos objetos de atribución.

El presente estudio busca aportar a los desafíos conceptuales y de medición relacionados con cómo los individuos se explican fenómenos ligados a la desigualdad socioeconómica, como son la riqueza y la pobreza. La pregunta que guió esta investigación fue: ¿se encuentran relacionadas las atribuciones de pobreza y riqueza? Si bien el foco de la investigación fue la confirmación estadística de un modelo conceptual de atribuciones internas y externas tanto para pobreza como riqueza, el análisis también posee implicancias para el desarrollo conceptual de la teoría de la atribución y los instrumentos de medición asociados. En la medida que se pueda confirmar la existencia de elementos comunes a la explicación de la riqueza y la pobreza, se podría también plantear que las atribuciones de distintos fenómenos asociados con la desigualdad social se encuentran relacionados y que, por lo tanto, se deberían estudiar en conjunto.

\section{Atribuciones de Riqueza y Pobreza}

El concepto de atribución es un objeto de estudio tradicional de la psicología social que se enfoca en entender cómo las personas explican el comportamiento de otros. Heider (1958) fue el primero en introducir la idea de inferencias causales relacionadas con el concepto de atribuciones, que corresponden a juicios acerca de los factores que podrían producir un efecto en una situación particular, y la distingue de las inferencias de rasgos (trait inferences) que asignan características particulares a individuos. Posteriormente, Kelley (1967) propone que, en condiciones de información deficiente, los individuos aplican esquemas que relacionan causas con efectos específicos, los que llevarían a explicar la conducta individual en función de características de individuos y/o de su entorno. La investigación en atribuciones ha enfatizado principalmente la distinción entre las causas internas y externas de la conducta, en particular a partir del planteamiento de la teoría del error fundamental de atribución (Ross, 1977), que sugiere que en las sociedades modernas existe una tendencia a privilegiar atribuciones internas o individuales por sobre las externas o contextuales. En su trabajo posterior, Weiner (1985) introdujo una conceptualización más compleja de las atribuciones, elaborando una taxonomía que distinguió entre tres dimensiones: locus (interna o externa), estabilidad (temporal o estable) y control (controlable o incontrolable). La presente investigación se centra en la dimensión de locus.

Desde estos aportes iniciales, la investigación en atribuciones representa un ámbito relevante y diverso en psicología social, siendo investigado en relación a variables como edad, género, etnicidad, raza, educación e ingresos (Hunt, 2004; Lepianka, Gelissen \& van Oorschot, 2010; McWha \& Carr, 2009; Mickelson \& Hazlett, 2014; Reutter et al., 2006; Sigelman, 2013; Wollie, 2009; Yeboah \& Kumi, 2012), experiencias personales de desventaja (Lepianka et al., 2010), ideología política (Bobbio, Canova \& Manganelli, 2010; Robinson, 2009), creencias sobre movilidad social (Bullock \& Waugh, 2005), creencias sobre desigualdades de ingresos y apoyo a políticas de bienestar (Bullock, Williams \& Limbert, 2003), conciencia de la pobreza (Kallio \& Niemelä, 2014), exposición a la pobreza (Reutter et al, 2006), valores psicosociales (Lepianka et al., 2010; Yeboah \& Kumi, 2012), salud mental (Mickelson \& Hazlett, 2014), emociones y comportamiento (Weiner, Osborne \& Rudolph, 2010) y actitudes hacia la pobreza (Cozzarelli, Wilkinson \& Tagler, 2001). Asimismo, es posible encontrar investigaciones que vinculan atribuciones con variables que van más allá de lo individual, tales como crecimiento económico, desempleo, regímenes de bienestar, gasto social (Kallio \& Niemelä, 2014; Yeboah \& Kumi, 2012) y niveles de desarrollo (da Costa \& Dias, 2014).

El estudio empírico sobre atribuciones de riqueza y pobreza se ha focalizado principalmente en la distinción de las dimensiones de locus y control de Weiner (1985) y comenzó con la propuesta de tres dimensiones de Feagin (1972), correspondientes a atribuciones individualistas, estructurales y fatalistas. En 
términos generales, las atribuciones individuales o internas enfatizan la responsabilidad personal en la situación de pobreza, las atribuciones estructurales, sociales o externas otorgan la responsabilidad a factores económicos y sociales externos al individuo, mientras que las atribuciones fatalistas destacan el rol de la suerte o el destino. En un estudio posterior, Feagin (1975) verificó este modelo de tres dimensiones en una muestra de Estados Unidos.

Desde las investigaciones de Feagin, es posible encontrar un número importante de estudios que han abordado el tema de las atribuciones, particularmente hacia la pobreza. En esta línea, diversos estudios empíricos han arribado al modelo de tres dimensiones, a través de técnicas multivariadas, como análisis de componentes principales y análisis factorial exploratorio (Feather, 1974; Hunt, 2004; Niemelä, 2008). Sin embargo, otros estudios han cuestionado la existencia unívoca de tres dimensiones, apoyando parcialmente el modelo de Feagin $(1972,1975)$. Además, investigaciones recientes identifican perfiles de atribución ambivalente, incluyendo simultáneamente atribuciones individuales y contextuales (Osborne \& Weiner, 2015).

Si bien los factores de atribuciones individualistas y estructurales poseen vasta evidencia a su favor, algunos autores discuten la presencia unívoca de un factor de atribuciones fatalistas, ya que este tiende a resultar en una combinación de ítems de tipo fatalista y estructural (Bullock, 1999; Bullock \& Limbert, 2003; Zucker \& Weiner, 1993) o a representar dimensiones híbridas que no corresponden ni a atribuciones estructurales ni a individuales (Bullock \& Limbert, 2003; Cozzarelli et al., 2001; Nilson, 1981). En estos estudios se señala que la dimensión fatalista no estuvo bien definida cuando se llevaron a cabo las técnicas de reducción de datos y, por lo tanto, no posee apoyo empírico sustantivo. Además, algunos autores indican que los ítems construidos para factores estructurales e individuales podrían abordar comportamientos lo suficientemente heterogéneos como para constituir dimensiones distintas o subdimensiones (Furnham, 1982; Lepianka et al., 2009; Morçöl, 1997; Payne \& Furnham, 1985).

En cuanto a las atribuciones de riqueza, los estudios empíricos en los que se ha abordado este tema son menos frecuentes y la mayoría se ha realizado en conjunto con atribuciones de pobreza. Los pocos estudios publicados en relación a riqueza no son concluyentes, ya que solo en uno de ellos se reprodujeron las tres dimensiones de Feagin (Hunt, 2004). Por otra parte, Forgas, Morris y Furnham (1982) realizaron un análisis factorial exploratorio y arribaron a un modelo de cuatro dimensiones, compuestas por el modelo clásico de atribuciones externas-sociales e internas-individuales, junto con otras dos nuevas dimensiones que denominaron antecedentes familiares y suerte-riesgo. Adicionalmente, Bullock et al. (2003) desecharon el modelo de Feagin y propusieron un modelo de cuatro factores distintos.

Los estudios que combinan tanto las atribuciones de pobreza como las de la riqueza de manera conjunta, además de ser escasos, son difíciles de comparar entre sí, dada la diferencia de instrumentos, operacionalizaciones y métodos. Por ejemplo, Stacey y Singer (1985) analizan por separado cuatro causas distintas entre sí para riqueza y pobreza en una muestra de 325 adolescentes, algo similar a lo realizado por Bullock y Limbert (2003), quienes extraen componentes principales distintos y por separado para cada objeto de atribución con datos de 131 estudiantes de pregrado. En otras investigaciones se intenta obtener tipos de atribución equivalentes para riqueza y pobreza. En esta línea, Hunt (2004), en base a una encuesta de 1.107 adultos, realiza un análisis factorial exploratorio para escalas de riqueza y pobreza por separado, pero extrayendo factores internos, externos y fatalistas para ambos constructos. En la misma línea, Bobbio et al. (2010) realizan un análisis de componentes principales para atribuciones tanto de pobreza como de riqueza por separado con 181 estudiantes universitarios, considerando dimensiones latentes similares (interna/externa) para ambos constructos. Finalmente, otro grupo de investigaciones se caracterizan por avanzar en la dirección confirmatoria de la estructura factorial de las atribuciones de riqueza y pobreza. Kluegel et al. (1995) encuentran buenos ajustes para un modelo confirmatorio de cinco dimensiones, correspondiente a atribuciones individuales y sociales de pobreza, atribuciones individuales y sociales de riqueza y una quinta dimensión compuesta por un ítem referido a deshonestidad. Por su parte, Kreidl (2000) analizó las respuestas a 14 ítems de atribuciones de riqueza y pobreza, concluyendo que, en el caso de pobreza, el modelo de tres factores (externas, internas y fatalistas) obtuvo buenos índices de ajuste, mientras que para las atribuciones de riqueza estableció un modelo de tres dimensiones, que denominó causas individuales, sistema y capital social.

Analizando la literatura existente, es posible sintetizar un conjunto de limitaciones y vacíos. En primer lugar, existe ausencia de una escala común y estable de indicadores que hayan sido utilizados transversalmente en los estudios de atribuciones de pobreza y riqueza. Esta situación implica que los diferentes modelos estimados no pueden ser comparados fácilmente, $\mathrm{y}$, por lo tanto, es difícil llegar a una 
conclusión razonable acerca de la confiabilidad y la validez de estas mediciones. En segundo lugar, no es posible identificar una dirección clara de la agenda de investigación en medición de atribuciones de riqueza y pobreza, ya que, si bien en algunos estudios como los de Hunt (2004) y Bobbio et al. (2010) se intenta extraer factores equivalentes para riqueza y pobreza, las investigaciones de Kluegel et al. (1995) y Kreidl (2000), orientadas hacia la confirmación de la estructura factorial, no son concluyentes respecto de factores comunes para ambos objetos de atribución. En este sentido, si bien la literatura revisada presenta evidencia de tipo confirmatoria para escalas de atribuciones, no existen antecedentes sobre estudios en los que se haya intentado asociar las atribuciones de pobreza con las de riqueza en términos de medición, sino que ellas siempre son analizadas como variables dependientes por separado. Si bien en algunos estudios se reportan correlaciones entre las variables, la pregunta sobre un posible modelo de medición común para ambos tipos de atribución — riqueza y pobreza - sigue abierta a discusión y verificación empírica.

En la presente investigación se apuntó a superar algunas de las limitaciones de medición de atribuciones de riqueza y pobreza mencionadas anteriormente. Para ello, se propuso la estimación de un modelo factorial confirmatorio, distinguiendo cuatro factores de atribuciones internas y externas, tanto para riqueza como pobreza, respectivamente, dado que estas dimensiones presentan mayor grado de evidencia empírica. Adicionalmente, se estimó una estructura de atribuciones similar, tanto para pobreza como riqueza, en la que la hipótesis fue que la atribución interna es un factor común tanto para pobreza como para riqueza, del mismo modo que la atribución externa. Este modelo de factores externos e internos comunes a distintos objetos es una derivación del modelo teórico general de atribución, que se encuentra presente a la base de algunas investigaciones anteriores, pero con algunas limitaciones en su operacionalización, como se señaló anteriormente. Para contrastar esta hipótesis se realizó una estimación de factores comunes a las causas de la riqueza y de la pobreza en un modelo factorial de segundo orden (Brown, 2006; Byrne \& Stewart, 2006; Marsh et al., 2009; Sayer \& Cumsille, 2001), el cual propone factores comunes como variables latentes a la base de otros factores latentes, en el marco de la estimación de un modelo confirmatorio basado en ecuaciones estructurales.

\section{Método}

\section{Instrumento}

En este estudio se utilizaron datos provenientes de la encuesta Justicia Social y Participación Ciudadana (Proyecto FONDECYT 11121203). Esta encuesta fue implementada en Chile durante el año 2013, en la que se preguntó por percepciones y creencias sobre la distribución de ingresos en Chile, junto con el grado de involucramiento en diferentes formas de participación política.

Las variables utilizadas consisten en una serie de 10 indicadores referidos a las causas de la pobreza y de la riqueza, basadas en la escala original de Feagin (1972) y en la versión presentada en la encuesta International Social Justice Project (ISJP) del año 2006 (ver https://www.sowi.hu-berlin.de/de/lehrbereiche/empisoz/forschung/archiv/isjp). Los ítems fueron traducidos al español y su comprensión fue evaluada en un piloto del cuestionario. Se incluyeron medidas sociodemográficas de sexo, edad, nivel educacional e ingreso.

El encabezado de esta sección de la encuesta fue: "En su opinión, ¿con qué frecuencia las siguientes razones podrían ser la causa de la pobreza [riqueza] en Chile?", seguido de cinco posibles causas para el caso de la pobreza (falta de habilidad, mala suerte, falta de esfuerzo, sistema económico y sistema educativo) y cinco causas homólogas para el caso de la riqueza (talento, suerte, trabajo duro, sistema económico y sistema educativo). Las respuestas se puntuaron en una escala de cinco opciones, de acuerdo a los siguientes valores: $1=$ nunca, $2=$ casi nunca, $3=$ a veces, $4=$ casi siempre, $5=$ siempre. .

Investigaciones previas con una versión similar de la escala del proyecto ISJP (2006) en Alemania han confirmado una estructura de dos factores para la escala de pobreza (atribuciones internas y externas), $\chi^{2}(5$, $N=3051)=50,88, p<0,001, \mathrm{CFI}=0,96, \mathrm{RMSEA}=0,06$ (Schneider \& Castillo, 2015)

\section{Participantes}

La muestra estuvo conformada por 1.245 participantes mayores de 18 años (mujeres $=714$ ) y abarcó las 88 comunas de Chile con más de 40.000 habitantes, excluyendo las regiones extremas del sur (Aisén y Magallanes), y con afijación para muestra en la Región Metropolitana (500 casos). 
El diseño muestral fue probabilístico estratificado por conglomerados, organizado en cuatro etapas de selección aleatoria (comunas, manzanas, hogares e individuos). La selección de las manzanas se realizó de manera proporcional al tamaño (PPT), según el número de viviendas y estratificando por región. La asignación de manzanas por región corresponde a su proporción de población urbana, con un promedio de cuatro manzanas por comuna. En las manzanas, luego las viviendas fueron seleccionadas por muestreo aleatorio simple, y de la misma manera los individuos al interior de los hogares.

El error muestral correspondió a un 2,8\% para un nivel de confianza de 95\%, calculado posteriormente a la recolección de los datos.

\section{Procedimiento}

El trabajo de campo de la encuesta se realizó entre agosto y septiembre, mediante entrevistas cara a cara en los hogares de los participantes, de aproximadamente 35 minutos de duración. Todos los participantes firmaron un consentimiento informado, el que contó con la aprobación del comité de ética de la Pontificia Universidad Católica de Chile.

\section{Análisis de Datos}

En primer lugar, se realizó un análisis descriptivo de las respuestas, con el objetivo de tener una mirada general de los datos y su distribución.

En segundo lugar, se abordó la pregunta por el modelo de medición de las atribuciones de riqueza y pobreza. El análisis de datos se realizó mediante análisis factorial confirmatorio, utilizando la librería lavaan de R (Rosseel, 2012). Los ítems fueron considerados categóricos ordenados, por lo que se utilizó el estimador mínimos cuadrados ponderados y varianza ajustada (weighted least squares means and variance adjusted, WLSMV). Si bien algunos factores se encuentran asociados a solo dos indicadores, la información total de la matriz de varianza-covarianza para el modelo completo permite que este se encuentre identificado, considerando que el primer ítem de cada factor se fija a tener peso igual a 1. El análisis comenzó con la estimación de un modelo de primer orden en el que se consideraron simultáneamente cuatro factores correspondientes a atribuciones externas e internas de pobreza y atribuciones externas e internas de riqueza. El paso siguiente fue estimar un modelo de segundo orden, en el cual las correlaciones entre los factores de primer orden fueron la base de construcción de un segundo modelo independiente (Marsh et al., 2009).

El ajuste del modelo a los datos se evaluó utilizando medidas globales de ajuste, como el estadístico $\chi^{2}$, junto con otras medidas menos sensibles al tamaño muestral, ya que en este caso es esperable que $\chi^{2}$ sea significativo en muestras mayores a $200 \mathrm{y}$, por tanto, signo de un mal ajuste. Concretamente, se utilizó el índice de Tucker-Lewis (TLI), el índice comparativo de Bentler-Bonett (CFI) y la raíz del error cuadrático medio de aproximación (RMSEA). Como criterios para establecer un buen ajuste de los modelos se consideró un $\chi^{2}$ no significativo ( $p>0,05$ ), un RMSEA inferior a 0,06 y valores superiores a 0,95 en el caso de CFI y TLI (Brown, 2006).

\section{Resultados}

En la Figura 1 se presentan los porcentajes por categorías generales de respuesta para los cinco ítems de atribuciones de pobreza y los cinco ítems de atribuciones de riqueza. En el gráfico se aprecia que los patrones de respuesta son variables en relación a cada indicador y que, además, no presentan una distribución que se agrupe en torno al promedio, lo cual indica que la estimación factorial debe ajustarse al carácter categórico ordenado de los indicadores. En general, se observa una tendencia a atribuir la pobreza a causas externas o estructurales (sistema económico y sistema educativo), por sobre las internas o individualistas (falta de habilidad, mala suerte y falta de esfuerzo), mientras que la riqueza se asocia una mezcla de atribuciones internas (talento, suerte y trabajo duro) y externas (sistema económico y sistema educativo). En el caso de la pobreza, las causas más atribuidas (i.e., que presentaron mayor porcentaje de respuesta tipo casi siempre y siempre) fueron sistema educativo y sistema económico, seguidos por falta de esfuerzo, falta de habilidad y mala suerte. Por otro lado, la causa más atribuida en relación a la riqueza fue trabajo duro, seguido por sistema económico, sistema educativo, talento y suerte. 
Frecuencia de razones que causan pobreza

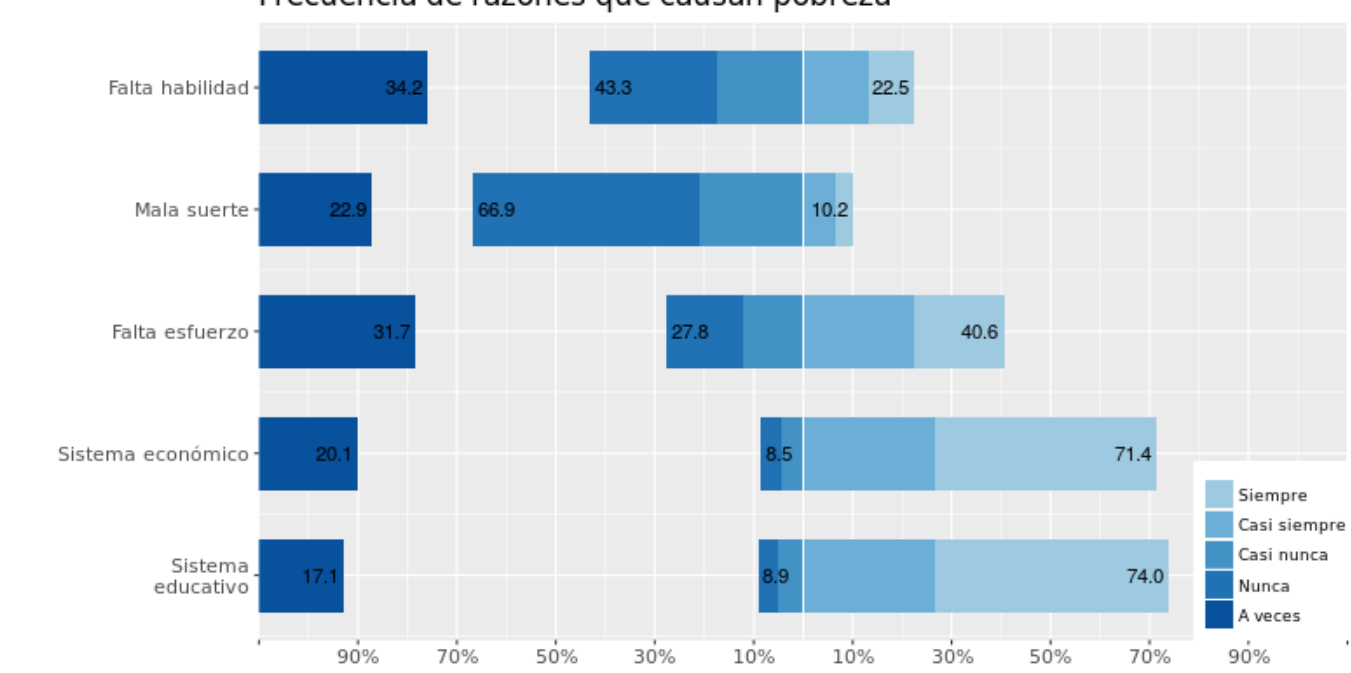

Frecuencia de razones que causan riqueza

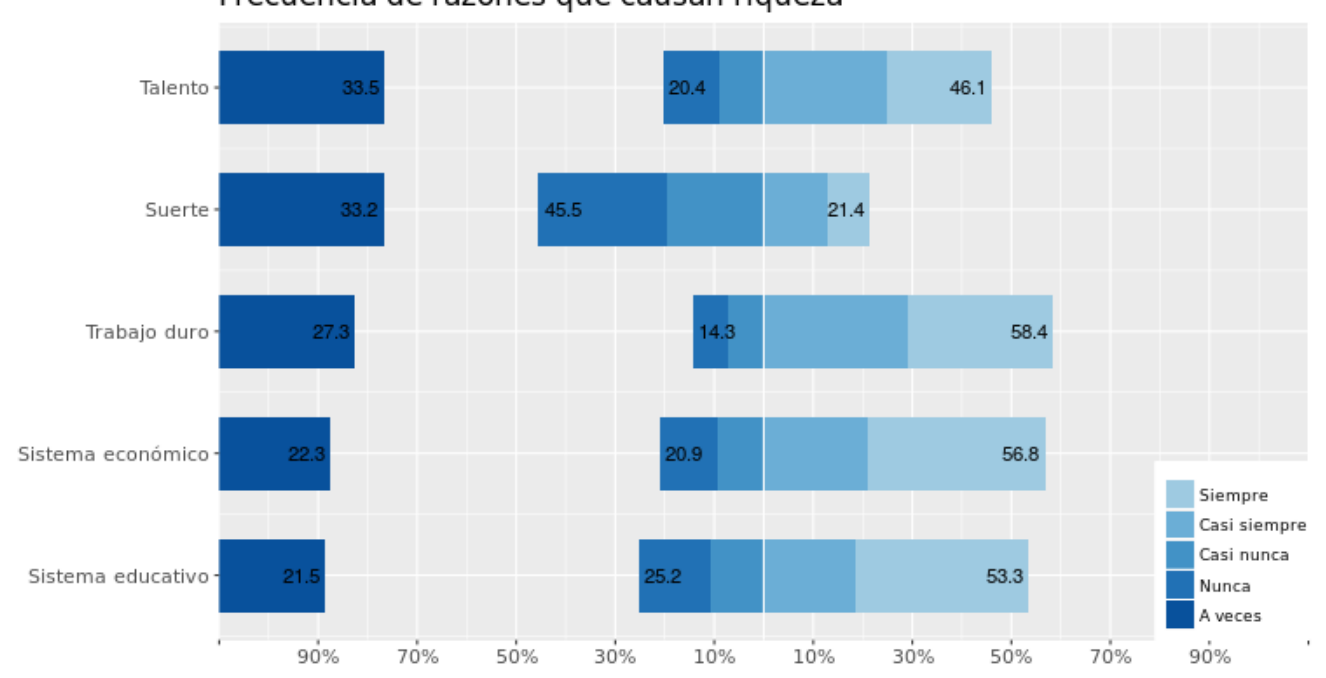

Figura 1. Porcentaje por categoría de respuesta para las atribuciones de riqueza y pobreza. Los porcentajes aparecen sumados para las categorías siempre y casi siempre, y para casi nunca y nunca

En la primera estimación del modelo de primer orden se observó un ajuste moderado, puesto que el valor de RMSEA se encuentra por sobre el rango convencional considerado aceptable, $\chi^{2}(29, N=1238)=347,69$, $p<0,001$, RMSEA $=0,095$, CFI $=0,955$, TLI $=0,930$.

En base al análisis de residuos de esta estimación, se constató que el ajuste se encontraba comprometido principalmente por una correlación no estimada entre los errores de los ítems referidos a Suerte en las atribuciones internas de riqueza y pobreza $(r=0,433, p<0,01)$. Dado que algunos estudios en el tema establecen la existencia de un factor de suerte o fatalista y que sería distinto a los factores internos y externos (Feagin, 1972; Feather, 1974; Hunt, 2004; Niemelä, 2008), se especificó una correlación entre los errores de estos indicadores. Esta modificación resultó en una mejora considerable de los niveles de ajuste, $\chi^{2}(28, N=1238)=135,29, p<0,001, \operatorname{RMSEA}=0,057, \mathrm{CFI}=0,985, \mathrm{TLI}=0,975$. El detalle de la estimación de parámetros se presenta en la Figura 2. En base a estos resultados y a los antecedentes de otras investigaciones, se optó por conservar la especificación con la correlación entre errores de los indicadores de suerte para la estimación del siguiente modelo. 


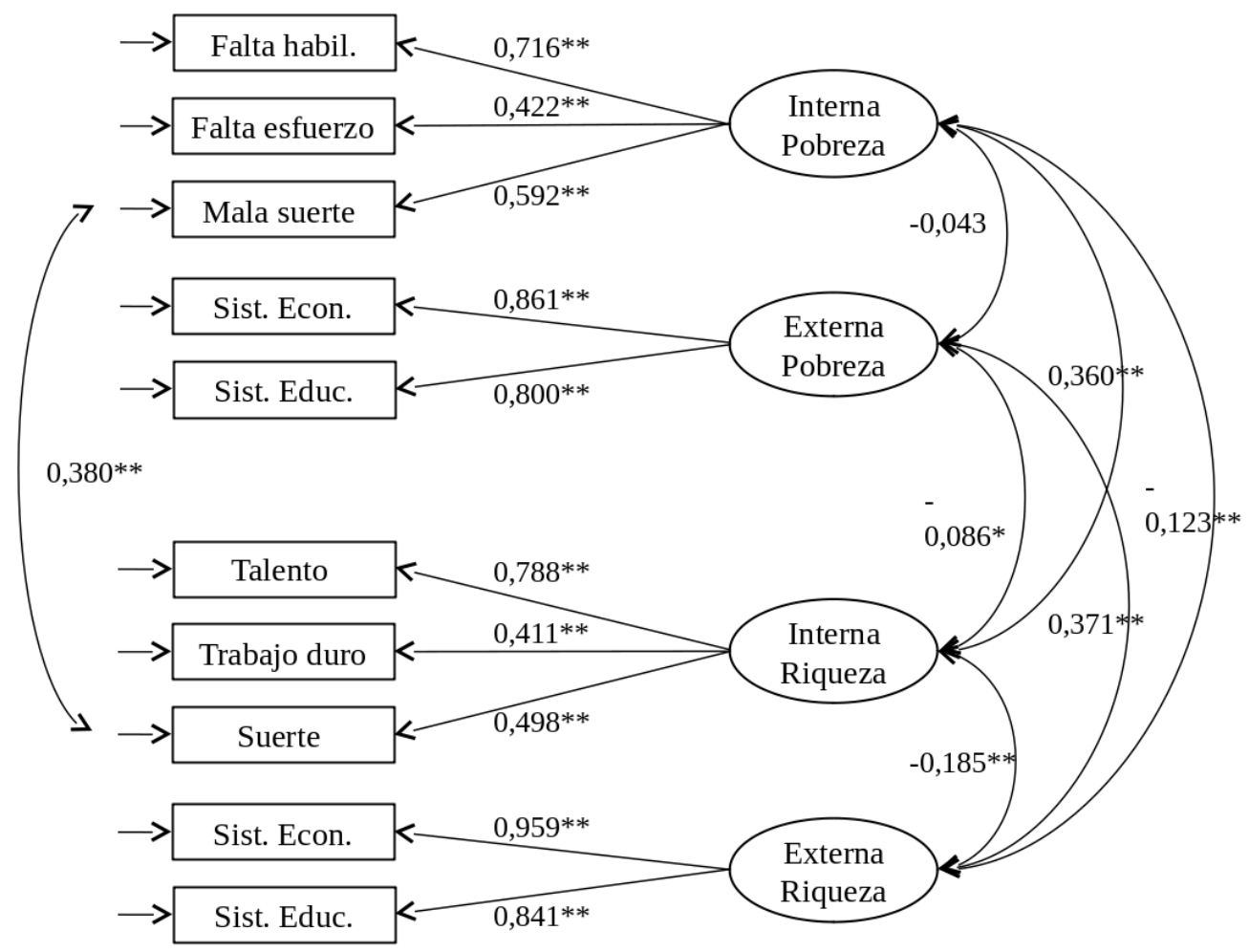

Modelo de Factores de Primer Orden

Estimador: Mínimos cuadrados ponderados y varianza ajustada (WLSMV) $\mathrm{N}=1238$

Figura 2. Modelo de atribuciones de riqueza y pobreza (factores de primer orden, solución estandarizada).

En la estimación del modelo de segundo orden se especificaron dos factores globales de atribuciones internas y externas, los cuales se exponen en la Figura 3, junto con los parámetros estimados. Este último modelo mostró buenos índices de ajuste, $\chi^{2}(29, N=1238)=133,52, p<0,001$, RMSEA = 0,055, CFI $=0,985$, $\mathrm{TLI}=0,975$, los que, en general, son superiores al modelo de primer orden y, además, generan una solución más parsimoniosa en términos sustantivos, al centrarse en la covarianza de dos variables latentes principales: atribución interna y atribución externa. Finalmente, es interesante notar que existe una correlación inversa moderada entre los factores de segundo orden $(r=-0,267, p<0,01)$, indicando una tendencia de que mayores atribuciones internas se asocian a menores externas, y viceversa. 


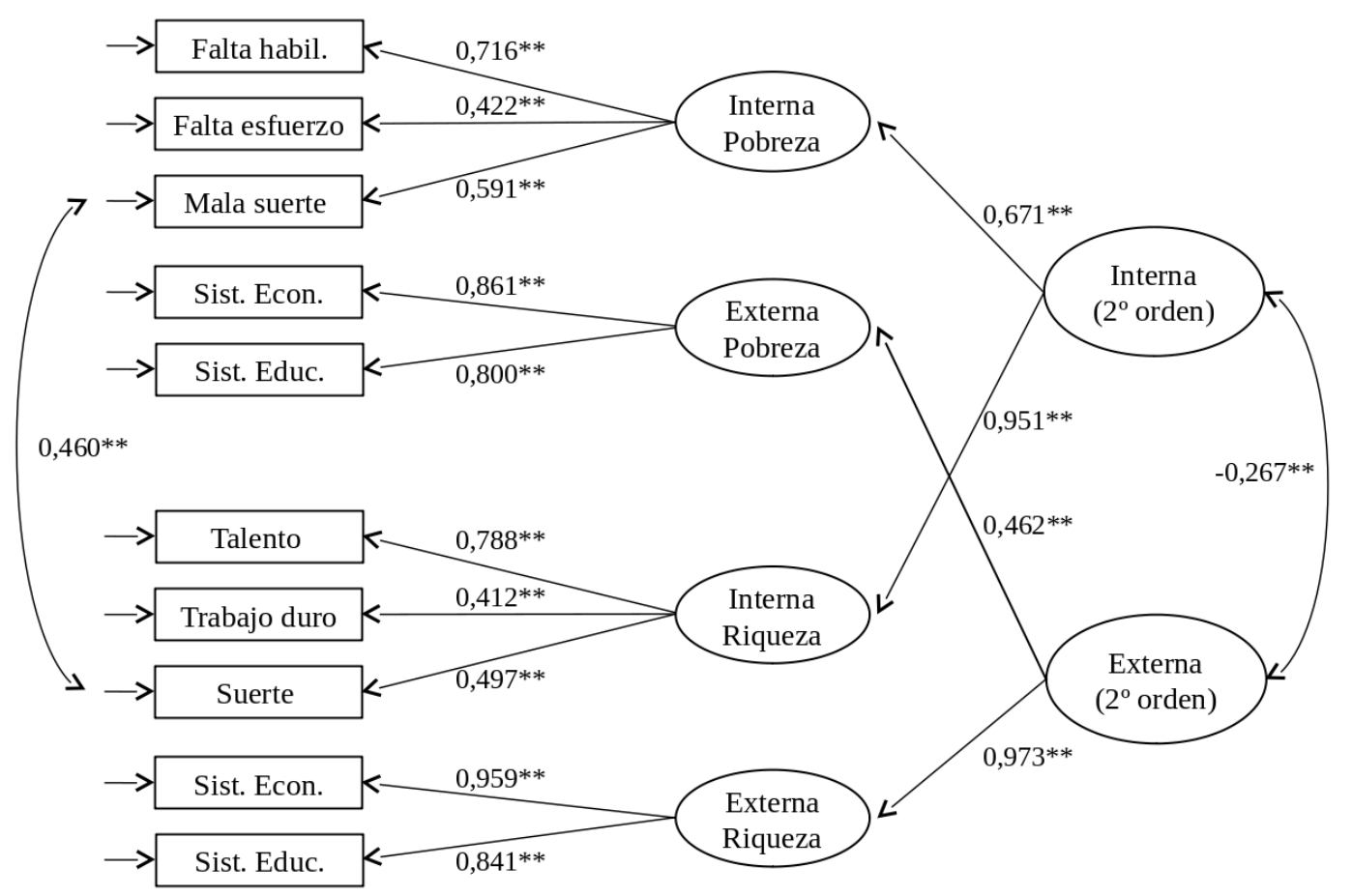

Modelo de Factores de Segundo Orden

Estimador: Mínimos cuadrados ponderados y varianza ajustada (WLSMV) $\mathrm{N}=1238$

Figura 3. Modelo de atribuciones de riqueza y pobreza (factores de segundo orden, solución estandarizada).

\section{Discusión}

Los principales resultados de esta investigación otorgan por primera vez evidencia confirmatoria en relación a la existencia de factores comunes de atribución interna y externa para riqueza y pobreza. En términos sustantivos, esto quiere decir que las atribuciones internas de la pobreza se asocian a atribuciones internas de la riqueza, ocurriendo lo mismo para las atribuciones externas. Esto permitiría plantear que individuos que atribuyan la pobreza a causas internas también tenderán a hacer lo mismo respecto de la riqueza, en un patrón atribucional genérico que tendería a la mantención del status quo. Además, se observó una relación inversa entre los factores de segundo orden del modelo, lo que podría sugerir que quienes tienden a explicar pobreza y riqueza basados en razones individualistas serían, en general, menos propensos a hacerlo en base a motivos sociales o externos.

Un hallazgo relevante del estudio lo constituye el rol de los indicadores de suerte. En propuestas originales del concepto de atribución, como la de Feagin (1972) y la de otros autores posteriores (Feather, 1974; Hunt, 2004; Niemelä, 2008), se establece un tercer factor relacionado con la suerte o el azar, elemento que aparece intermitentemente en la literatura o bien se confunde con otros factores (Bullock, 1999; Bullock \& Limbert, 2003; Zucker \& Weiner, 1993). En el presente estudio, el ajuste del modelo mejora considerablemente al estimar un parámetro que asocia indicadores referidos a suerte en ambas temáticas (riqueza y pobreza). Por lo tanto, futuros estudios se podrían beneficiar de la inclusión de un número mayor de indicadores referidos a la suerte, que sean equivalentes a los internos y externos, para poder realizar una estimación más precisa de este patrón atribucional. Además, sería deseable contar con un mayor número de indicadores por dimensión para estimar independientemente la calidad de cada factor, así como también contar con un modelo que abarque otras dimensiones de la atribución, además del locus, como son la controlabilidad y la estabilidad en la perspectiva de Weiner (1985).

Entre las limitaciones del estudio, la escala de atribuciones puede ser corregida homologando de manera más estricta el contenido de las afirmaciones de atribución para riqueza y para pobreza. Por ejemplo, en esta 
escala se considera habilidad para pobreza y talento para riqueza, así como falta de esfuerzo para pobreza y trabajo duro para riqueza. Una versión más depurada de la escala debería incluir los mismos conceptos para ambos tipos de atribución, para evitar posibles fuentes de error asociadas a la interpretación por parte de los entrevistados. Por otro lado, la estimación realizada no permite afirmar que el modelo funciona de la misma manera en distintos grupos, por ejemplo, en personas de distinto nivel socioeconómico. Para superar esta limitación se requieren análisis de equivalencia del modelo en distintos subgrupos, lo que será abordado en un estudio posterior.

En cuanto a posibilidades de investigación futura, un aspecto relevante es establecer en qué medida el modelo atribucional de segundo orden se replica en otras sociedades. Para ello, la investigación comparativa internacional es clave, así como también la estimación de invarianza métrica del modelo. Además, la estimación factorial confirmatoria hasta ahora permite visibilizar correlaciones lineales entre variables, pero queda abierta la pregunta respecto de la existencia de tipos o clases de individuos que manifiestan distintos patrones de atribución. Por ejemplo, se podría hipotetizar la existencia de individuos que atribuyen predominantemente de manera interna, otros externa y otros con una combinación de ambas. Finalmente, a partir de la estimación presentada en este artículo se puede avanzar en relacionar otras características individuales con patrones atribucionales, tales como nivel socioeconómico, ideología política, estatus, preferencias políticas, actitudes hacia la distribución, entre otras.

\section{Referencias}

Bobbio, A., Canova, L. \& Manganelli, A. M. (2010). Conservative ideology, economic conservatism, and causal attributions for poverty and wealth. Current Psychology, 29, 222-234. https://doi.org/10.1007/s12144-010-9086-6

Brown, T. A. (2006). Confirmatory factor analysis for applied research. New York, NY: Guilford Press.

Bullock, H. E. (1999). Attributions for poverty: A comparison of middle-class and welfare recipient attitudes. Journal of Applied Social Psychology, 29, 2059-2082. https://doi.org/10.1111/j.1559-1816.1999.tb02295.x

Bullock, H. E. \& Limbert, W. M. (2003). Scaling the socioeconomic ladder: Low-income women's perceptions of class status and opportunity. Journal of Social Issues, 59, 693-709. https://doi.org/10.1046/j.0022-4537.2003.00085.x

Bullock, H. E. \& Waugh, I. M. (2005). Beliefs about poverty and opportunity among Mexican immigrant farm workers. Journal of Applied Social Psychology, 35, 1132-1149. https://doi.org/10.1111/j.1559-1816.2005.tb02163.x

Bullock, H. E., Williams, W. R. \& Limbert, W. M. (2003). Predicting support for welfare policies: The impact of attributions and beliefs about inequality. Journal of Poverty, 7(3), 35-56. https://doi.org/10.1300/J134v07n03_03

Byrne, B. M. \& Stewart, S. M. (2006). The MACS approach to testing for multigroup invariance of a second-order structure: A walk through the process. Structural Equation Modeling, 13, 287-321. https://doi.org/10.1207/s15328007sem1302_7

Cozzarelli, C., Wilkinson, A. V. \& Tagler, M. J. (2001). Attitudes toward the poor and attributions for poverty. Journal of Social Issues, 57, 207-227. https://doi.org/10.1111/0022-4537.00209

da Costa, L. P. \& Dias, J. G. (2014). Perceptions of poverty attributions in Europe: A multilevel mixture model approach. Quality \& Quantity, 48, 1409-1419. https://doi.org/10.1007/s11135-013-9843-3

Feagin, J. R. (1972). Poverty: We still believe that God helps those who help themselves. Psychology Today, 6, 101-110, 129.

Feagin, J. R. (1975). Subordinating the poor: Welfare and American beliefs. Englewood Cliffs, NJ: Prentice-Hall.

Feather, N. T. (1974). Explanations of poverty in Australian and American samples: The person, society, or fate? Australian Journal of Psychology, 26, 199-216. https://doi.org/10.1080/00049537408255231

Forgas, J. P., Morris, S. L. \& Furnham, A. (1982). Lay explanations of wealth: Attributions for economic success. Journal of Applied Social Psychology, 12, 381-397. https://doi.org/10.1111/j.1559-1816.1982.tb00873.x

Furnham, A. (1982). Why are the poor always with us? Explanations for poverty in Britain. British Journal of Social Psychology, 21, 311-322. https://doi.org/10.1111/j.2044-8309.1982.tb00553.x

Heider, F. (1958). The psychology of interpersonal relations. Hillsdale, NJ: Lawrence Erlbaum.

Hunt, M. O. (2004). Race/ethnicity and beliefs about wealth and poverty. Social Science Quarterly, 85, 827-853. https://doi.org/10.1111/j.0038-4941.2004.00247.x

Kallio, J. \& Niemelä, M. (2014). Who blames the poor? Multilevel evidence of support for and determinants of individualistic explanation of poverty in Europe. European Societies, 16, 112-135. https://doi.org/10.1080/14616696.2013.787435

Kelley, H. H. (1967). Attribution theory in social psychology. Nebraska Symposium on Motivation, 15, 192-238.

Kluegel, J. R., Csepeli, G., Kolosi, T., Örkény, A. \& Neményi, M. (1995). Accounting for the rich and the poor: Existential justice in comparative perspective. En J. R. Kluegel, D. S. Mason \& B. Wegener (Eds.), Social justice and political change: Public opinions in capitalist and post-communist States (pp. 179-207). New York, NY: De Gruyter.

Kreidl, M. (2000). Perceptions of poverty and wealth in western and post-communist countries. Social Justice Research, 13, 151-176. https://doi.org/10.1023/A:1007597807110

Lepianka, D., Gelissen, J. \& van Oorschot, W. (2010). Popular explanations of poverty in Europe: Effects of contextual and individual characteristics across 28 European countries. Acta Sociologica, 53, 53-72. https://doi.org/10.1177/0001699309357842

Lepianka, D., van Oorschot, W. \& Gelissen, J. (2009). Popular explanations of poverty: A critical discussion of empirical research. Journal of Social Policy, 38, 421-438. https://doi.org/10.1017/S0047279409003092

Marsh, H. W., Muthén, B., Asparouhov, T., Lüdtke, O., Robitzsch, A., Morin, A. J. S. \& Trautwein, U. (2009). Exploratory structural equation modeling, integrating CFA and EFA: Application to students' evaluations of university teaching. Structural Equation Modeling, 16, 439-476. https://doi.org/10.1080/10705510903008220

McWha, I. \& Carr, S. C. (2009). Images of poverty and attributions for poverty: Does higher education moderate the linkage? International Journal of Nonprofit and Voluntary Sector Marketing, 14, 101-109. https://doi.org/10.1002/nvsm.336 
Mickelson, K. D. \& Hazlett, E. (2014). "Why me?": Low-income women's poverty attributions, mental health, and social class perceptions. Sex Roles, 71, 319-332. https://doi.org/10.1007/s11199-014-0414-4

Morçöl, G. (1997). Lay explanations for poverty in Turkey and their determinants. The Journal of Social Psychology, 137, 728-738. https://doi.org/10.1080/00224549709595494

Niemelä, M. (2008). Perceptions of the causes of poverty in Finland. Acta Sociologica, 51, 23-40. https://doi.org/10.1177/0001699307086816

Nilson, L. B. (1981). Reconsidering ideological lines: Beliefs about poverty in America. The Sociological Quarterly, 22, 531-548. https://doi.org/10.1111/j.1533-8525.1981.tb00679.x

Osborne, D. \& Weiner, B. (2015). A latent profile analysis of attributions for poverty: Identifying response patterns underlying people's willingness to help the poor. Personality and Individual Differences, 85, 149-154. https://doi.org/10.1016/j.paid.2015.05.007

Payne, M. \& Furnham, A. (1985). Explaining the causes of poverty in the West Indies: A cross-cultural comparison. Journal of Economic Psychology, 6, 215-229. https://doi.org/10.1016/0167-4870(85)90010-8

Reutter, L. I., Veenstra, G., Stewart, M. J., Raphael, D., Love, R., Makwarimba, E. \& McMurray, S. (2006). Public attributions for poverty in Canada. Canadian Review of Sociology, 43, 1-22. https://doi.org/10.1111/j.1755-618X.2006.tb00852.x

Robinson, J. W. (2009). American poverty cause beliefs and structured inequality legitimation. Sociological Spectrum, 29 , $489-518$. https://doi.org/10.1080/02732170902904681

Ross, L. (1977). The intuitive psychologist and his shortcomings: Distortions in the attribution process. En L. Berkowitz (Ed.), Advances in experimental social psychology, Volume 10 (pp. 173-220). New York, NY: Academic Press. https://doi.org/10.1016/S0065-2601(08)60357-3

Rosseel, Y. (2012). lavaan: An R package for structural equation modeling. Journal of Statistical Software, 48(2), 1-36. https://doi.org/10.18637/jss.v048.i02

Sayer, A. G. \& Cumsille, P. E. (2001). Second-order latent growth models. En L. M. Collins \& A. G. Sayer (Eds.), New methods for the analysis of change (pp.179-200). Washington, DC: American Psychological Association.

Schneider, S. M. \& Castillo, J. C. (2015). Poverty attributions and the perceived justice of income inequality: A comparison of East and West Germany. Social Psychology Quarterly, 78, 263-282. https://doi.org/10.1177/0190272515589298

Sigelman, C. K. (2012). Rich man, poor man: Developmental differences in attributions and perceptions. Journal of Experimental Child Psychology, 113, 415-429. https://doi.org/10.1016/j.jecp.2012.06.011

Sigelman, C. K. (2013). Age differences in perceptions of rich and poor people: Is it skill or luck? Social Development, 22, 1-18. https://doi.org/10.1111/sode. 12000

Stacey, B. G. \& Singer, M. S. (1985). The perception of poverty and wealth among teenagers. Journal of Adolescence, 8, 231-241. https://doi.org/10.1016/S0140-1971(85)80055-5

Weiner, B. (1985). An attributional theory of achievement motivation and emotion. Psychological Review, 92, 548-573. https://doi.org/10.1037/0033-295X.92.4.548

Weiner, B., Osborne, D. \& Rudolph, U. (2011). An attributional analysis of reactions to poverty: The political ideology of the giver and the perceived morality of the receiver. Personality and Social Psychology Review, 15, 199-213. https://doi.org/10.1177/1088868310387615

Wollie, C. W. (2009). Causal attributions for poverty among youths in Bahir Dar, Amhara Region, Ethiopia. Journal of Social, Evolutionary, and Cultural Psychology, 3, 251-272. https://doi.org/10.1037/h0099319

Yeboah, S. A. \& Kumi, E. (2012). Attributions for poverty: A survey of student's perception. International Review of Management and Marketing, 2, 83-91. Extraído de https://www.econjournals.com/index.php/irmm/article/view/169/pdf

Zucker, G. S. \& Weiner, B. (1993). Conservatism and perceptions of poverty: An attributional analysis. Journal of Applied Social Psychology, 23, 925-943. https://doi.org/10.1111/j.1559-1816.1993.tb01014.x

Fecha de recepción: Enero de 2017.

Fecha de aceptación: Marzo de 2018. 